Aus der Universitäts-Augenklinik zu Göttingen.

(Direktor: Geheimrat Prof. Dr. v. Hippel.)

\title{
Über den Nutzen des Neu-Tuberkulins (Bacillenemulsion) bei der Tuberkulose des Anges.
}

\author{
Von
}

Dr. med. Hermann Davids, früher Assistent der Klinik, jetzt Augenarzt in Münster i. W.

Mehrfache Anfragen, die von verschiedenen Kliniken und Ärzten bezüglich der Kochschen Tuberkulinpräparate an die Göttinger Universitäts-Augenklinik gerichtet wurden, sowie Unklarheiten, die ich in der Literatur fand, veranlassen mich, bevor ich zu meinem eigentlichen Thema komme, auf die verschiedenen Präparate etwas näher einzugehen. Die Anfragen liessen erkennen, dass sowohl in bezug auf die Art des Tuberkulins als auch vornehmlich in bezug auf die Dosierung der verschiedenen Präparate Unklarkeiten und Irrtümer herrschten. Dies ist begreiflich, da zu einer erfolgreichen Anwendung des Tuberkulins zunächst eine gewisse Erfahrung gehört, die an einem grösseren Material leicht gewonnen werden kann, die aber an vereinzelten Fällen, wie sie sich dem praktischen Augenarzt bieten, schwer zu erlangen ist. Dazu kommt noch, dass die ersten Gebrauchsanweisungen, die dem Tuberkulin beigegeben wurden, insofern zu Irrtümern Veranlassung gaben, als von Koch selbst in diesen Anweisungen stets von Grammen und Milligrammen gesprochen wurde, wodurch Ärzte und Apotheker zu der falschen Annahme kamen, dass sich die Gebrauchsanweisungen auf die feste Substanz des Tuberkulins bezogen. Diese sollte aber für die Dosierung gleichgültig sein. Daher sind die Gebrauchsanweisungen für Alt-Tuberkulin später in der Weise geändert, dass in ihnen nur mehr von Kubikcentimetern usw. der Originalflüssigkeit die Rede ist. Auch die neven Gebrauchsanweisungen für das Tuberkulin T. R. beziehen sich nicht mehr auf die feste Substanz, sondern auf Kubikcentimeter usw. der Originalflüssigkeit. Diese Änderung ist nun meines Erachtens 
nicht genügend bekannt geworden, was ich aus verschiedenen Anfragen an die Göttinger Augenklinik schliesse.

Aufmerksam machen möchte ich an dieser Stello auch auf die Berichtigung, die Ruppel(22) kürzlich bezüglich des Tuberkulins T. R. veröffentlichte. Aus dieser erfahren wir, dass in $1 \mathrm{ccm}$ Tuberkulin T.R. gar nicht $10 \mathrm{mg}$ fester Substanz enthalten sind, wie allgemein angenommen werden musste. Die von Koch selbst gemachte Angabe ist vielmehr so zu verstehen, dass $1 \mathrm{ccm}$ T.R. nur die immunisierende Substanz von $10 \mathrm{mg}$ getrockneten Tuberkelbacillen enthält. Der Trockengehalt selbst beträgt in $1 \mathrm{ccm}$ nur $0,002 \mathrm{~g}$ !

Da es nun durchaus notwendig ist, dass man sich bei der Anwendung des Tuberkulins ganz klar ist über die Art und die Dosierung des Präparates, so will ich an dieser Stelle kurz das Wichtigste uiber die Entstehung, die Anwendung und die Dosierung der drei verschiedenen Präparate mitteilen. Die allgemeinen Regeln, die für alle drei Arten Tuberkulin gleich sind und seit langer Zeit fest stehen, sollen hier nicht berỉcksichtigt werden, vielmehr werde ich mich auf Mitteilung dessen beschränken, was zur Vermeidung von Irrtümern zu wissen notwendig ist. Bei den nachfolgenden Ausführungen halte ich mich fast wörtlich an die Originalangaben der Höchster Farbwerke, die mir bereitwilligst zur Verfügung gestellt wurden, und füge, wo es mir wichtig erscheint, die Erfahrungen hinzu, die in der Göttinger Augenklinik gesammelt wurden.

Die drei Präparate, die Koch für die Tuberkulose empfahl, sind: das Alt-Tuberkulin, das Neu-Tuberkulin (Tuberkulin T. R.) und das Neu-Tuberkulin (Bacillenemulsion).

1. Das Alt-Tuberkulin, 1890 durch Koch bekannt gegeben, wird durch Eindampfen von Tuberkulosekulturen bei mässiger Temperatur gewonnen. Nachdem die Kulturflüssigkeiten auf $1 / 10$ ihres Volumens konzentriert sind, werden die Bacillen durch Filtration entfernt. Durch das Eindampfen der Kulturen.wird nicht nur eine Konzentration der Flüssigkeit, sondern auch eine gewisse Extraktion der Bacillenleiber erreicht, wodurch der Gehalt des Tuberkulins an wirksamer Substanz nicht unwesentlich erhöht wird.

$\mathrm{Da}$ das Alt-Tuberkulin in der Augenheilkunde wohl nur noch zu diagnostischen $Z_{\text {wecken }}$ verwandt wird, will ich nur hierauf eingehen. Nach der neuesten Vorschrift nimmt man zur ersten Einspritzung $0,0001 \mathrm{ccm}$ bis $0,001 \mathrm{ccm}$ der Originalflüssigkeit; die Höhe der Dosis richtet sich nach dem Alter und dem Kräftezustand der Patienten. 
Beträgt die erste Dosis $0,001 \mathrm{~cm}$, so steigt man, wenn keine Reaktion erfolgt, nach zwei Tagen auf das Doppelte und darauf auf $0,005 \mathrm{ccm}$. Erfolgt auch jetzt keine Reaktion, so steigt man auf $0,01 \mathrm{ccm}$.

An der Göttinger Universitäts-Augenklinik nahmen wir zuerst bei Erwachsenen $0,001 \mathrm{ccm}$, stiegen sodann auf 0,002 resp. auf $0,003 \mathrm{ccm}$ und gaben als höchste Dosis 0,005 ; mit dieser Dosierung erreichten wir sichere Resultate (vgl. Krankengeschichten). Bei Kindern wurden je nach Alter entsprechend geringere Dosen angewandt.

Als Verdünnungsflüssigkeit wählt man bei dem Alt-Tuberkulin $1 / 2 \%$ ige Karbolsäurelösung.

2. Das Neu-Tuberkulin (Tuberkulin T.R.) wurde von Koch im Jahre 1897 empfohlen. Dieses Präparat sollte die immunisierenden Eigenschaften des Tuberkulins besitzen, ohne aber heftige Allgemeinreaktionen hervorzurufen, wie sie beim Alt-Tuberkulin oft beobachtet wurden. Gewonnen wird das Tuberkulin T.R., indem zunächst den fein zerriebenen Tuberkelbacillen alle lösliohen Stoffe mit destilliertem Wasser entzogen werden. Diese Lösung, von Koch das T. O. genannt, enthält die toxischen Bestandteile der Bacillen, während der unlösliche Tuberkelbacillenrückstand, der nur geringe toxische Wirkung auf tuberkulöse Individuen besitzt, die Gesamtmenge der in den Tuberkelbacillen vorhandenen immunisierenden Substanzen umfasst. Dieser Rückstand wird durch successives, scharfes Trocknen, Zerreiben, Aufnehmen von Wasser und durch Zentrifugieren bei mehrmaliger Wiederholung dieser Operation in eine Reihe von Lösungen überführt, die vereinigt werden und für den Organismus gut resorbicrbar sind. Die Anfangsdosis des T.R. soll nach den neuesten Vorschriften im allgemeinen $1 / 5000 \mathrm{~cm}=0,0002 \mathrm{ccm}$ der Originalflüssigkeit betragen.

Diese Dosis ist dieselbe, die von Hippel(2) im Jahre 1904 angab, nur musste damals infolge der anders lautenden Vorschriften auch die Bezeichnung anders sein. Wenn von Hippel nach langen Versuchen die Anfangsdosis auf ${ }^{1 / 500} \mathrm{mg}$ Trockensubstanz festsetzte, so ist das nur eine andere Bezeichnung für denselben Wert, denn $1_{500} \mathrm{mg}$ Trockensubstanz nach der alten Berechnung ist gleich $1 / 5000 \mathrm{ccm}$ Originalfüssigkeit. In Göttingen stiegen wir bei der Behandlung fast nie über $1 \mathrm{mg}$ Trockensubstanz $={ }_{1}^{1}{ }_{10} \mathrm{ccm}$ der Originalflüssigkeit, da wir bei höheren Dosen durchaus keine besseren Resultate erzielten. Als Verdünnungsflüssigkeit wählt man beim T. R. eine $20 \%$ ige Glycerinlösung. 
3. Die Bacillenemulsion, von uns kurz nur so genannt, um Verwechslungen mit dem Neu-Tuberkulin T. R. zu vermeiden, wurde von Koch im Jahre 1901 bekannt gegeben. Sie stellt eine Aufschwemmung pulverisierter Tuberkelbacillen in Wasser mit Zusatz gleicher Teile Glycerin dar. Die Bacillenemulsion entspricht also einer Mischung ron T. R. und T. O. Nach Kochs Vorschrift beginnt man die Injoltionen mit dem 2000. Teil des Präparates, was $0,0025 \mathrm{mg}$ Bacillensubstanz bedeutet, denn in $1 \mathrm{ccm}$ der Bacillenemulsion befinden sich $5 \mathrm{mg}$ der pulverisierten Tuberkelbacillen.

In Göttingen begannen wir mit emer Dosis von 1/1000 resp. $1_{500} \mathrm{mg}$ Trockensubstanz (vgl. Krankengeschichten) und stiegen in bekannter Weise bis 1, höchstens 11/2 mg. Bei dieser Dosierung erhielten wir vorzügliche Resultate.

Die Verdünnungen werden mit $0,8 \% \mathrm{NaCl}$-Lösung, oder wenn sie mehrere Tage konserviert werden sollen, mit $0,8 \% \mathrm{NaCl}$ - und $0,5 \%$ Phenollösung hergestellt.

Ich hoffe durch diese kurze Zusammenstellung eine leichte Orientierung über die drei Tuberkulinpräparate von Koch gegeben zu haben.

Die Literatur über Anwendung von Tuberkulin bei der Tuberkulose der Augen ist nicht gross. v. Hippel (2, 12) war der erste, der systematische Versuche mit dem Kochschen Tuberkulin bei der Augentuberkulose anstellte und eine Methode begründete, die noch heute an der Göttinger Augenklinik unverändert geübt wird und von vielen Ärzten übernommen wurde. $\quad$. Hippel wandte bei den ersten Fällen das Alt-Tuberkulin an. Nach Bekanntgabe des T.R. ging er zu diesem Präparat über und veröffentlichte 1904 die vorzüglichen Resultate, die er selbst bei schwerster Tuberkulose des Auges mit dem T.R. erzielt hatte. Nach dieser Arbeit v. Hippels sind von verschiedenen Seiten die Erfahrungen mitgeteilt, die auf diesem Gebiete gemacht wurden. Wenn diese Mitteilungen auch nicht sehr zahlreich sind, so genügen sie doch, um die Überzeugung zu gewinnen, dass das Tuberkulin sich in vielen Fällen als ein ausserordentlich wertvolles Mittel bei der Behandlung der Augentuberkulose bewährt hat. Die Arbeiten befassen sich meist mit dem Neu-Tuberkulin T. R. Günstige Resultate wurden vor allem bei der Tuberkulose der Iris und der Cornea gewonnen, während das Tuberkulin nach Ansicht verschiedener Augenärzte bei der Tuberkulose der Bindehaut ungeeignet sein soll.

Ich glaube, die Resultate würden im allgemeinen noch günstiger 
ausgefallen sein, wenn bei der Anwendung des Tuberkulins die Vorschriften immer genauer beachtet wären. Vor allem ist mir aufgefallen, dass in verschiedenen Fällen viel zu wenig Injektionen gemacht wurden. Man kann von einer abgekürzten Tuberkulinkur nach unserer Erfahrung keinen vollen Erfolg erwarten, vor allem wird man in solchen Fällen viele Rezidive erleben. Sowohl von Patienten als auch vom Arzt muss viel Geduld geübt werden, wenn gute und bleibende Resultate erlangt werden sollen (vgl. Krankengeschichten).

Sehr warm für das Tuberkulin T.R. und für die v. Hippelsche Methode tritt Stock(11) ein. Er glaubt mit von Hippel in dem T. R. (bei richtiger Anwendung!) ein Mittel empfehlen zu können, das chronische Augenerkrankungen, die bis jetzt trotz Behandlung immer schlechter wurden, zur Heilung bringt. Er empfiehlt die Hippelsche Dosierung. Nach seiner Ansicht ist das T. R. das weitaus beste und sicherste Mittel, das wir zur Behandlung der tuberkulösen Iridocyclitis besitzen; Stock hebt hierbei besonders die rasche Aufhellung der Medien hervor und die hierdurch bedingte Besserung des Sehvermögens. Geringe Aussichten auf Erreichung eines normalen Visus haben nach Stock die Patienten mit tuberkulöser Chorioiditis dissem. wegen der irreparablen Schädigung der Stäbchen und Zapfen.

Gar keine Angaben fand ich in der Literatur über die Anwendung der Bacillenemulsion bei der Tuberkulose der Augen. Es ist dies um so auffallender, als Koch selbst diesem Präparat besondere Vorzüge nachrühnt, und als auch von andern Seiten tatsächlich sehr gute Erfolge, zum Beispiel bei der Behandlung der Lungentuberkulose, emielt wurden. Mir scheint, als wenn dieses letzte Präparat Kochs unter den Augenärzten überhaupt noch nicht den gebührenden Platz erlangt hat, was ich nicht nur aus dem Fehlen von Angaben in der Literatur, sonderu auch aus Anfragen, die an die Göttinger Augenklinik gerichtet wurden, schliessen muss. Möglich ist übrigens, dass in einigen Veröffentlichungen, in denen kurz von Neu-Tuberkulin die Rede war, die Bacillenemulsion gemeint wurde. Wie dem auch sei, Angaben, aus denen die Anwendung dieses Präparates bei der Behandlung der Tuberkulose der Augen deutlich hervorging, fand ich nicht.

In Göttingen wurde die Bacillenemulsion von uns seit Anfang 1906 angewandt und zwar, wie wir unten sehen werden, mit dem besten Erfolg. Rezidive, die v. Hippel nach der Behandlung mit Neu-Tuberkulin T.R. beobachtete, veranlassten ihn, diese 
Tatsache Koch mitzuteilen. Koch schlug darauf im Februar 1906 vor, Versuche mit einem verbesserten Präparat anzustellen. Dieses Tuberkulin, die sogenannte Bacillenemulsion, umfasst nach Koch die gesamte Masse der Tuberkelbacillen, so dass bei ihm alles zur Geltung kommt, was im stande ist, Antikörper zu bilden. Nach den Erfahrungen Kochs erreicht man mit diesem Präparat dauerhaftere Erfolge als mit den alten Präparaten.

Seit dieser ermutigenden Mitteilung Kochs wurde die Bacillenemulsion bei der Tuberkulose der Augen in der Universitäts-Augenklinik zu Göttingen dauernd benutzt. Um ein objektives Urteil über die Anwendung und Wirkung dieses Mittels zu ermöglichen, will ich die betreffenden Krankengeschichten kuxz mitteilen.

\section{Fall.}

Ernst K., 23 Jahre alt. Familienanamnese belanglos. Patient selbst litt als Kind an Tuberkulose der Knochen, seit zehn Tagen sind Blendungserscheinungen vor dem rechten Auge aufgetreten.

Am 28. VIII. 06 erfolgt Aufnahme in die Klinik. Der innere Befund ergibt nichts besonderes, an Händen und Armen grosse, alte Narben von Knochenerkrankungen herrührend. Rechtes Ange mittelstark gerötet. An der Descemet unten einzelne sehr feine Beschläge, im Kammerfalz unten und etwas nach innen ein kleiner, gelber Knoten yon ungefäbr Stecknadelkopfgrösse. Iris hyperämisch. Pupille auf Atropin weit, unten anssen eine schmale, hintere Synechie. Auf der vorderen Linsenkapsel Reste abgerissener Synechien. Medien klar. Papille normal, über den ganzen Fundus verteilt alte, chorioiditische Herde, z. T. stark pigmentiert. L. A. chorioiditische Veränderungen wie rechts. R. S. $=$ fast 1,0, L. S. $=1,0$.

6. IX. 06. Wurde mit Atropin und Umschlägen behandelt. Heute ist ein zweiter Knoten vom selben Aussehen innen unten sichtbar geworden.

8. IX. Wegen Verdacht anf Tuberkulose Probeinjektion von $0,001 \mathrm{ccm}$ Alt-Tuberkulin.

Am 9. IX. allgemeine und lokale Reaktion.

13. IX. Fühlt sich wieder wohl, Auge wieder blasser. Aussen unten ist im Kammerfalz ein dritter Knoten entstanden. Ord. 1/500 $\mathrm{mg}$ Bacillenemulsion.

25. IX. Da Patient nach $1_{500} \mathrm{mg}$ einmal Kopfsehmerzen hatte, wird mit den Injektionen langsam gestiegen, heute ${ }^{4} / 500 \mathrm{mg}$ Bacillenemulsion. Das rechte Auge ist inzwischen blasser geworden, nur unten ist es noch etwas gerötet. Die Knötchen dagegen sind eher grösser als kleiner.

5. X. Bulbus blass. Knötehen verkleinern sich etwas.

16. $X$. Hin und wieder noch geringe Injektion.

25. X. Ange dauernd reizlos. Der äussere Knoten ist nur noeh eben sichtbar.

1כ. XI. 1. Injektion von $1 \mathrm{mg}$ Bacillenemulsion. Injektionen in letater Zeit gut vertragen. Patient fühlt sich wohl. 
Vom 15. bis 26. XI. dauernd $1 \mathrm{mg}$. Da Reaktion auftrat, wird auf $1 / 2 \mathrm{mg}$ zurückgegangen.

5. XII. Patient entlassen, erhielt im ganzen 40 Injektionen. Allgemeinbefinden ist sehr gut, Patient hat an Körpergewicht bedeutend zugenommen. Beide Augen in letater Zeit dauernd reizlos und blass. R. A. Cornea klar, unten an der Descemet noch einige dunkle Beschläge. Kammerwasser klar. Pupille rund, eng, zentral, reagiert prompt. Iris nicht mehr hyperämisch. Die drei Knoten baben sich deutlich verkleinert, sind noch als kleine, flache, gelbliche Prominenzen siehtbar. Die Beschläge auf der vorderen Linsenkapsel sind unverändert, ebenso die Synechie. Auch die Aderhautveränderungen zeigen denselben Befund. L. A. unverändert. Beiderseits $\mathrm{S} .=1,0$.

19. XII. Vorstellung. Befund unverändert. Patient ist arbeitsfähig. (Die Knoten im Kammerfalz bilden sich später vollständig zurïck, vgl. S. 250).

\section{Fall.}

Paula W., 30 Jahre alt. Litt seit acht Jahren an Entzündungen des linken Auges, die sich vor einem Jahre versehlimmerten. Trotz ärztlicher Behandlung wurde das Sehen immer schlechter. Sonst war die Frau gesund. Keine hereditäre Belastung.

Bei der Aufnahme am 18. XII. 06 war der Befund folgender:

R. A. normal. L. A. Bulbus reizlos. Cornea spiegelnd; im oberen Teil wie auch unten in ihr eine mässig grosse, rauchige Trübung. Unten, aussen und oben einzelne, peripher gelegene, gelbliche, dicke Beschläge an der Descemet. Einer von ihnen ist fast stecknadelkopfgross. Iris stark schmutzig verfärbt, geschwollen und wie mit einem sulzigen Exsudat überlagert. Unten im Kammerfalz ein Knötchen, kleinhanfkorngross, graugelblich von sulzigem Aussehen, das nach hinten ins Irisgewebe abergeht. Aussen ron diesem ein ebensolches ron Stecknadelkopfgrösse, ein gleiches oben aussen im Kammerfalz. Gefässe .sind anf ihnen nicht zu erkennen. Pupille auf Atropin wenig erweitert. In der hinteren Corticalis feine Trübungen. Glaskörper diffus getrübt. Papille daher undeutlich sichtbar. L. S. $=$ Finger in $3 \mathrm{~m}$. R. A. normal. $\mathrm{S}$. $=1,0$.

19. XII. 06. $0,001 \mathrm{cem}$ Alt-Tuberkulin.

20. XII. Kopfschmerzen. Temp. 38,8 .

21. XII. Patientin fühlt sich wohl. Kein Fieber mehr.

22. XII. 1/1000 ing Bacillenemulsion.

8. I. 07. Steigende Dosen werden reaktionslos vertragen, heute $8 / 1000 \mathrm{mg}$. Auge dauernd reizlos. Cornealtrübungen und Beschläge unverändert. Der Knoten oben aussen schrumpft stark zusammen, auch die unteren bilden sich zurück. Um letztere liegt jetzt ein sulziges Exsudat.

29. I. L. S. Finger in $5 \mathrm{~m}$. Patientin erhält $9 / 100 \mathrm{mg}$.

30. III. Patientin reagierte auf höhere Dosen als ein $1 / 10 \mathrm{mg}$ wiederholt mit Kopfschmerzen and lokalen Erseheinungen wie Trübung der Cornea und des Kammerwassers. Nach ganz allmählichem Steigen wurde schliesslich $4 / 10 \mathrm{mg}$ gut vertragen.

5. IV. Entlassung, Patientin erhielt 50 Injektionen. R. A. normal. I. A. reizlos. Cornea spiegelnd; oben und unten die erwähnten, rauchigen Trïbungen. Die Beschläge sind noch zum grossen 'Teil sichtbar. Das 
Knötehen oben aussen ist völlig zurüekgebildet, die unten im Kammerfalz sind zu einem stecknadelkopfgrossen Knötehen verselimolzen und von grauem Aussehen; das Exsudat, das sie umgab, ist versehwunden. Die Regenbogenhaut ist atrophisch, Linse unverändert. Kammerwasser klar. Glaskörper noch diffus getrübt, doch so anfgehellt, dass man Papille und Gefässe gut erkennen kann. L. S. 0,1 Ord. Schonung, Vorstellung nach viex Wochen.

30. V. Vorstellung. Bulbus blass. Kammerwasser klar. Von den Knötchen ist nichts mehr zu sehen. Iris atrophiseh. Fundusbild etwas klarer. L. S. $=0,1$.

12. VII. Vorstellung: Auge dauernd reizlos. Cornea spiegelnd, ist völlig klar, ebenso das Kammerwasser; Iris reizlos, atrophisch. Linse unverändert, Glaskörper noch getrübt, doch gutes Fundusbild erhältlich. Nirgends Knötchen zu sehen. L. S. 0,1.

18. X. Befund unverändert.

\section{Fall.}

Julius S., 14 Jahre alt. Patient war angeblich vorher immer gesund. Seit Sommer 1906 ist das linke Auge entzündet, aueh hat das Sehvermögen seitdem abgenommen. Der Zustand verschlimmerte sich trotz ärztlicher Behandlung.

Am 26. XI. 06 erfolgt Aufnahme in die Klinik. R. A. normal. L. A. Bulbus mittelstark conjunetival etwas mehr ciliar injiziert. In der Cornea parac. unten ein den tiefsten Schichten angehöriges, kreisrundes, gelbliches Infiltrat von einem Durchmesser von ungefähr $11 / 2 \mathrm{~mm}$. Zwei weitere Infiltrate liegen im oberen Drittel der Hornhaut, eins oben innen, ein anderes oben aussen; beide sind grösser als das erst beschriebene, auch etwas dichter, gehören aber ebenfalls den tiefsten Schichten an. Tiefliegende, pinselförmige Gefässe ziehen reichlich von oben zu den beiden letzten Infiltraten. Hinter dem oben innen gelegenen liegt ein dicker, gelber Beschlag an der Descemet. Im Kammerfalz und zwar in der unteren Hälfte liegen vier grauweisse Prominenzen, zwei grössere, flache - Basis 2-3 mm - und zwei stecknadelkopfgrosse. Kammerwasser klar. Vorderkammer normal tief. Iris stark hyperämisch und etwas geschwollen. Auf der inneren Hälfte derselben sieht man zwei deutliche, besonders stark vaskularisierte Buckel von ungefähr $3 \mathrm{~mm}$ Durchmesser, zwei ähnliche, nicht so grosse befinden sich im äusseren Teil der Iris. Die Pupille hat sich auf Atropin uur nach aussen oben erweitert; Papillarrand sonst breit verwachsen. Auf der vorderen Linsenkapsel liegen mehrere braune Beschläge und ein graues Exsudat. Vom Fundus ist nur mattroter Reflex erhältlich. Der Bulbus ist nicht druckempfindlich. R. S. $=1,0$. L. S. $=$ Finger in $3 \mathrm{~m}$.

4. XII. 06. Nach der 2. Injektion von $0,003 \mathrm{ecm}$ Alt-Tuberkulin heftige allgemeine und lokale Reaktion.

6. XII. Erscheinungen geschwunden. Temperatur normal. Bulbus blasser wie bei der Aufnahme.

7. XII. 1/500 $\mathrm{mg}$ Bacillenemulsion.

18. XII. Da einmal allgemeine Reaktion auftrat, wird sehr langsam gestiegen, hente ${ }^{4} / 500$ mg. L. A. Bulbus aussen blass. Die beiden Infiltrate oben in der Cornea gehen jetzt diffus ineinander uber und sind von zahl- 
reichen Gefässen durchzogen. Kammerwasser klar, der gelbe Beschlag ist versehwunden. Dagegen hat sich einer der Knoten innen im Kammerfalz etwas vergrössert. Die Irisbuckel zeigen sehr starke, netzartige Vaskularisation.

21. XII. 6/500 $\mathrm{mg}$.

9. I. 07. Trotzdem nicht gestiegen wurde, traten doch zweimal Temperatursteigerungen mit Kopfschmerzen auf. L. A. Innen leicht conjunetival, stärker ciliar gerötet. Neben der Hornhaut innen ein kleiner gelber, episkleraler Buckel, von einem Gefässring umgeben. Die besehriebenen Infiltrate sind im Abheilen begriffen. Unten in der Cornea ist ein kleines frisches Infiltrat entstanden. Im Kammerfalz sind noch drei ganz flache Prominenzen sichtbar. Die Irisbuckel sind ebenfalls flacher, ihre Vaskularisation ist geringer. Das Pupillarexsudat ist dünner.

23. I. Es wurde sehr langsam gestiegen, heute ${ }^{9} / 500 \mathrm{mg}$. Injektionen werden jetzt gut vertragen.

26. II. Da wieder allgemeine und lokale Reaktionen auftraten, musste sehr langsam bis $4 / 50 \mathrm{mg}$ gestiegen werden, am 24 . II. wurde wieder auf ${ }^{2} / 50 \mathrm{mg}$ zurückgegangen, diese Dosis wurde gut vertragen. L. A. Innen hart am Limbus ist der kleine, gelbe Knoten wenig verändert. Die Infiltrate sind abgeheilt. Von den Knötchen im Kammerfalz ist nur noch eins innen unten angedeutet; hier hat sich ein zartes Exsudat an die Hornhaut gelegt. Die Buckel der Iris sind versehwunden. Iris ist nicht mehr hyperämisch. Pupillarexsudat bedeutend dünner. L. S. $=: 0,2-0,3$.

24. III. Trotzdem in grossen Zwischenräumen und langsam steigend injiziert wurde, trat doch einmal wieder allgemeine Reaktion und lokale um den episkleralen, kleinen Knoten auf. Daher Rïckgang auf $1 / 50 \mathrm{mg}$.

30 . III. Gestern nach Injektion von ${ }^{3} 5_{50} \mathrm{mg}$ wieder allgemeine Reaktion. Der kleine episklerale Knoten scheint sich etwas vergrössert zu haben.

Am 25. IV. L. S. $=0,3$. Iridektomie (das gewonnene Irisstïckchen wird in die Vorderkammer eines Kaninchens gebracht, wo es reaktionslos liegen bleibt).

Wegen der häufigen Reaktionen nach der Bacillenemulsion wurde bei den letzten 5 Injektionen T. R. verwandt. Trotz sehr geringer Dosen trat auch bei $T$. R. einmal allgemeine Reaktion auf.

6. V. Patient erhielt 41 Injektionen Bacillenemulsion, 5 Injektionen T. R. Patient sieht viel wohler aus als bei der Aufnahme und hat erheblich an Körpergewicht zugenommen. Der Entlassungsbefund ist folgender: R. A. normal. L. A. reizlos. Innen hart am Limbus ist das episklerale Knötchen kleiner und geringer vaskularisiert. In der Hornhant liegt parazentral unten eine stecknadelkopfgrosse etwas dichtere Trübung, oben innen eine grössere zarte, die von feinen Gefässehen durchzogen ist, sodann oben anssen eine ganz zarte, nur noch mit der Lupe deutlich sichtbare Trübung. Kammerwasser klar, Vorderkammer normal tief. Iris frei von entzïndlichen Erseheinungen. Iris nirgends mehr vorgebuckelt. Keine Knoten mehr im Kammerfalz. Gestalt der Pupille unverändert. Pupillarexsudat stark verdünnt. Ziemlich deutliches normales Fundusbild. L. $\mathrm{S} .=0,3$ Ord Schonung.

25. V. Vorstellung. L. A. reizlos. Befund unverändert. 


\section{Fall.}

Frau H., 24 Jahre alt. Stammt aus gesunder Familie. Hat selbst angeblich zweimal Blutsturz gehabt. War als Kind von acht Jahren augenleidend, im 19. Lebensjahr abermalige Entzündung. Wurde damals erst 1/2 Jahr zu Hause, dann vierzehn Tage in der Klinik wegen Iritis und Keratitis behandelt. War später wieder mehrere Wochen in der Klinik wegen Episkleritis, Keratitis und Seclusio.

Am 25. IV. 06. Wiederaufnahme in die Klinik mit folgendem Befund: R. A. reizlos. In der Hornhaut alte Trübungen. Aussen verläuft neben ihr in der Sklera eine ungefähr $2 \mathrm{~mm}$ breite, bläulichschwarze Zone. Vorderkammer normal. Oben innen ein mittelbreites Colobom, zahlreiche hintere Synechien. Fundus deutlich, normal. L. A. leicht gerötet. Aussen neben der Cornea ein ähnlicher, bläxlicher Bezirk der Sklera als rechts. Oben aussen in der Hornhaut eine Trübung, die von zarten Gefässen durchzogen wird. Diese Trübung gehört den tiefsten Schichten der Cornea an, ist sehr dicht und erstreckt sich zungenförmig bis ins Pupillargebiet; sie macht ganz den Eindruck eines Knotens, der in der Hornhaut liegt. Sonst befinden sich in der Hornhaut noch alte Randtrübungen. An der Descemet kleine, braune Beschläge. Vorderkammer normal tief. Iris hyperämisch. Pupillarrand durch breite Synechien fixiert. Feines Pupillarexsudat. Mittelbreites Colobom nach oben. Vom Fundus mattroter Reflex, kein Bild. Tonus herabgesetzt. R. S. $=0,5-0,6$. L. S. $=$ Finger in $2 \mathrm{~m}$.

26. IV. Wegen Verdacht anf Tuberkulose $0,001 \mathrm{~cm}$ Alt-Tuberkulin.

27. IV. Temperatur 40,1. Patientin fühlt sich schwer krank. L. A. stärker gerötet.

29. IV. Erscheinungen geschwunden. Aufnahmebefund.

30. IV. I/500 $\mathrm{mg}$ Bacillenemulsion.

10. V. Da einmal leichte Temperatursteigerung auftrat, wird sehr langsam gestiegen, heùte erhält Patientin ${ }^{4} /_{500} \mathrm{mg}$. Die Injektion des linken Auges ist nur noch sehr gering. Der Knoten in der Hornhaut bildet sich von der medialen Seite zurijck. Die Iris ist kaum noch hyperämisch.

25. V. Injektionen werden gut vertragen, gestern $1 / 100 \mathrm{mg}$. L. A. blass. Pupillarexsudat aussen oben resorbiert. Tonus fast normal. L. S. $=0,4$.

29. VI. Injektionen werden gut vertragen, heute $1 \mathrm{mg}$. Linkes Auge dauernd reizlos. Der Hornhautknoten hat sich immer mehr zurüekgebildet, ist unten schon gut durchscheinend. Vom Pupillarexsudat sind unten nur noch Reste sichtbar. L. S. $=0,6-0,7$.

2. VIII. Augapfel dauernd blass, Hornhautknoten so weit zurüekgebildet, dass nur noch eine hauchartige Trübung besteht. Der 'Tonus ist völlig normal. Seit dem 29. VI. erhält Patientin ständig $1 \mathrm{mg}$, das gut vertragen wird.

13. VIII. Nach 54 Injektionen wird Patientin heute entlassen. R. A. zeigt den Befund der Aufnahme. L. A. völlig reizlos, die erwähnte, dunkle Skleralzone ist unverändert. An Stelle des Knotens oben aussen sieht man nur noch eine ganz feine Trübung, die von wenigen kleinen Gefässen durchzogen wird. Die übrigen Trübungen der Hornhaut sind unverändert. Die Vorderkammer normal tief, Kammerwasser klar, Beschläge resorbiert. Colo- 
bom und Pupillargebiet frei, nur innen unten ist noch ein kleiner Rest des Exsudats sichtbar. Iris nicht mehr hyperämisch. Fundus normal, ebenso der Tonus. R. S. $=0,6$. L. $\mathrm{S} .=0,7$ bis fast 0,8 . Ord. Sehonung. 13. XI. Vorstellung. Befund unverändert.

1. VIII. 07. Hornhauttrübung oben aussen noch lichter. Rest des Pupillarexsudat dünner. Sonst Befund unverändert. R. S. $=0,6$. L. S. $=0,7-0,8$.

\section{Fall.}

Christine E., 25 Jahre alt, wurde schon wiederholt wegen Chorioiditis dissem. und Keratitis prof. behandelt. Keine Tuberkulose in der Familie.

12. XI. 06. Wegen frischer Entzündnng Wiederaufnahme in die Klinik. R. A. Cornea klar bis auf einige alte Randtrubungen. Pupille rund, mehrere hintere Synechien. Altes Exsudat im Pupillargebiet mit zentralem Loch. Alte Chorioiditis dissem. L. A. Bulbus leicht gerötet. Im episkleralen Gewebe, medial von der Comea 2 stecknadelkopfgrosse, glasige Knötchen, um die herum feine, neugebildete Gefässe ziehen. Die Conjunctiva über ihnen ist verschieblich. Unten innen an der Corneoskleralgrenze ein kleiner Buckel, in dessen Umgebung die Hornhaut getrübt ist. Unten liegen in den tiefsten Schichten der Cormea mehrere kleinste Infiltrate, zu denen Gefässe ziehen. Vorderkammer normal tief. Iris etwas hyperämisch. Pupille auf Atropin mittelweit. Auf der vorderen Linsenkapsel Reste eines alten Exsudats. Zahlreiche alte, stark pigmentierte, chorioiditische Herde. R. S. $=0,2-0,3$. L. S. = Finger in $5 \mathrm{~m}$.

15. XI. Wegen Verdacht auf Tuberkulose Probeinjektion.

20. XI. Nach der dritten Injektion (0,005 $\mathrm{cem}$ Alt-Tuberkulin) deutliche allgemeine und lokale Reaktionen.

22. XI. Erscheinungen geschwunden. Unten sind kleine, neve Skleralbuckel entstanden, sonst besteht der Aufnahmebefund. Es wird mit der Injektion von 1/1000 $\mathrm{mg}$ Bacillenemulsion begonnen.

2. XII. Bulbus immer noch etwas gerötet. Unten am Limbus ist ein frisehes, tiefliegendes Infiltrat entstanden. Es wird mit den Dosen sehr langsam gestiegen.

28. XII. Heute $1 / 100 \mathrm{mg}$. Injektionen werden gut vertragen. Bulbus fast reizlos. Skleralknötchen fast alle verschwunden.

7. I. 07 . Infiltrate heilen jetzt anch ab.

5. II. Erhält $1 \mathrm{mg}$, das Auge ist reizlos.

28. III. Entlassung. $1 \mathrm{mg}$ wurde dauernd gut vertragen. L. A. völlig reizlos, keine Knötchen mehr auf der Sklera. Infiltrate abgeheilt. In den tiefsten Schichten der Cornea verschiedene lichte Trübungen. Kammerwasser klar. Auf der Linsenkapsel die erwähnte Veränderung. Iris reizlos. Pupille mittelweit, rund, Fundus unverändert. L. S. $=$ Finger in $5 \mathrm{~m}$. R. A. unverändert. Patientin erhielt im ganzen 60 Injektionen.

19. IV. Vorstellung. Befund unverändert.

24. VIII. Vorstellung. Keine Veränderung.

$$
\text { 6. Fall. }
$$

Marie K., 35 Jahre alt. War schon als Kind augenleidend, wurde wiederholt wegen Tuberkulose der Hornhaut behandelt, 2 mal mit sehr gutem 
Erfolg mit T. R, doch stellten sich Rezidive ein. Wegen frischer Entzündung seit 3 Monaten erfolgt am 16. II. 06 Wiederaufnahme in die Klinik. R. A. mässig stark gerötet. Cornea im ganzen mittelstark getrübt. Von oben ziehen kleine Gefässe in die Hornhaut. An der Descemet nnten zahlreiche, feine Beschläge, oben aussen drei grosse, gelbe. Kammerwasser trüb. Pupille auf Atropin etwas erweitert. Iris etwas hyperämisch, unten eine langgezogene, hintere Synechie. L. A. reizlos, alte dichte Hornhauttrübung. R. S. $=0,1$. L. S. $=0,2$.

17. II. 07. Zunächst wiederum T. R., mit $\left.1 / 500 \mathrm{mg}^{1}\right)$ beginnend.

2. III. Injektionen werden gut vertragen. Bulbus blass. Kammerwasser klar; die feinen Beschläge haben abgenommen.

13. III. Feine Beschläge resorbiert, die dicken oben werden kleiner.

31. III. Injektionen gut vertragen, hente $1 / 50 \mathrm{mg}$. Bulbus dauernd reizlos. Die dicken Beschläge sind kaum noch sichtbar. Iris noch leicht hyperämisch.

20. IV. Da am 19. IV. nach $4 / 5 \mathrm{mg}$ allgemeine und lokale Reaktion auftrat, wird auf $1 / 5 \mathrm{mg}$ zurüekgegangen. R. A. reizlos. In der Hornhaut, den tiefsten Schichten angehörig, eine mitteldichte, streifige Trübung, die breit oben am Rande beginnt und bis über das Pupillargebiet herabzieht. Sie wird von feinen Gefässen durehzogen. Die Beschläge sind sämtlich resorbiert. Das Kammerwasser ist klar. Iris noch immer leicht hyperämiseh, Synechie unverändert. R.S. $=0,3-0,4$. L. A. unverändert, S. $=0,1-0,2$. 24. IV. 1/5 $\mathrm{mg}$ T. R.

26. IV. Da nach T. R. einmal eine ziemlich stärke Reaktion auftrat, wird heute versuchsweise $\left.{ }^{1}\right|_{10} \mathrm{mg}$ Baeillenemulsion injiziert.

27. IV. Injektion ist gut vertragen, es wird daher in gewohnter Weise gestiegen.

8. V. 7/10 $\mathrm{mg}$ Bacillenemulsion.

9. V. Bacillenemulsion wird vorzüglich vertragen. Auch in höherer Dosis wie das T. R., bei dem über $1 / 5 \mathrm{mg}$ nicht gestiegen werden durfte. Patientin wird entlassen mit folgendem Befund: L. A. unverändert. R. A. reizlos. Trübung der Hornhaut wie unterm 20. IV. beschrieben, jedoch ist sie von unten und von den Seiten lichter geworden, oben ist sie noch am dichtesten. Hier ziehen auch noch einige tiefe, feine Gefässe in die Trübung hinein. Keine Beschläge an der Descemet. Kammerwasser klar. Iris völlig reizlos. Pupille auf Atropin erweitert, Synechie erhalten. Undentliches Fundusbild. R. S. $0,3-0,4$. L. S. $=0,1-0,2$.

17. VI. Vorstellung. Cornealtrübung hat sich weiter aufgehellt. R. S. $=0,5-0,6$.

3. VI. 07. Vorstellung: L. A. unverändert. R. A. dauernd reizlos. Trübungen von den Seiten noch lichter geworden. R. S. $=0,6$.

\section{Fall.}

Gustav R., 14 Jahre alt. Patient wurde wiederholt wegen skroful. Keratitis behandelt und zwar 5 mal klinisch. In letzter Zeit war das L. A. trotz dauernder Behandlung nie ganz reizlos.

1) Vgl. S. 233 . 
Am 16. VII. 06. Wiederaufnahme. R. A. normal. L. A. Lidränder gerötet. Starke Lichtscheu und Epiphora. Bulbus mittelstark gerötet. Im Zentrum der Hornhaut eine alte, grosse Trübung, durch die die Pupille völlig verdeckt wird. In dieser Trübung erkennt man mehrere kleine, tiefliegende Infiltrate. Von allen Seiten ziehen zahlreiche, oberflächliche and tiefgelegene Gefässe durch die Hornhant. Pupille trotz Atropin eng. Visus nicht zu bestimmen.

18. VII. Da bei der Hartnäckigkeit des Leidens an Tuberkulose gedacht werden muss, wird eine Probeinjektion von $0,001 \mathrm{ccm}$ Alt-Tuberkulin gemacht.

19. VII. Starke allgemeine und lokale Reaktion.

25. VII. Patient fuhlt sich wohl. Befund wie bei der Aufnahme. 1/1000 mg Bacillenemulsion.

12. VIII. Langsam steigende Injektionen werden gut vertragen, heute $1 / 100 \mathrm{mg}$. Der Bulbus ist allmählich blass geworden. Die Cornealgefässe befinden sich in Rüekbildung, die Infiltrate heilen $a b$.

31. VIII. $1 / 10 \mathrm{mg}$. Injektionen dauernd gut vertragen. Auge dauernd reizlos. Hornhaut hellt sich anf.

7. IX. Da hölere Dosen sehlecht vertragen werden, wird wieder auf $1 / 10 \mathrm{mg}$ zuriekgogangen.

26. IX. Es ist bis ${ }^{3} / 10 \mathrm{mg}$ gestiegen. Patient fühlt sich sehr wohl, hat 3 Pfd. zugenommen. Das Auge ist dauernd reizlos. Die Gefässe sind zurückgebildet, die Infiltrate abgeheilt. Hornhaut hellt sich weiter auf, Pupille bleibt auf Atropin weit.

24. X. Ohne sonstige Reaktion entstand ein zarter Pannus von oben, der sich jedoch unter steigenden Injektionen wieder zurückbildete. Heute ${ }_{110} \mathrm{mg}$. Auge reizlos. L. $\mathrm{S}$. = Finger in $2-3 \mathrm{~m}$.

10. XI. In poliklinische Behandlung entlassen. Patient erhielt 50 Injektionen, als höehste Dosis $9 / 10 \mathrm{mg}$. Der Entlassungsbefund ist folgender: R. A. unverändert. Linkes Auge völlig reizlos. In der Hornhant eine mitteldichte, tiefgelegene, zentrale Trübung von der Grösse einer mittelweiten Pupille. Dieselbe ist unregelmässig begrenzt. Die Hornhaut rings herum ist leicht wolkig getrübt. Ganz vereinzelte, in Rückbildung begriffene, oberflächliche Gefässe ziehen in die Hornhaut hinein. Kammerwasser klar. Pupille auf Atropion weit. L. S. $=0,1$.

15. XI. Vorstellung. Befund unverändert.

25. XI. Bulbus oben aussen leicht gerötet. Ord. Atropin, Verband. 27. XI. Kleiner Pannus oben aussen.

7. XII. Pannus heilt ab.

14. XII. Geheilt entlassen.

9. V. 07. Auf Erkundigung teilt Patient mit, dass es ihm sehr gut geht.

\section{Fall,}

Heinrich H., 8 Jahre alt. Stammt aus tuberkulös belasteter Familie. Vater litt an Bluthusten. Die Mutter ist gesund. Keine Anhaltspunkte für Lues. Seit mehreren Wochen ist das rechte Auge entzündet, trotz ärztlicher Behandlung keine Besserung.

23. III. 07. Untersuchung in der medizinischen Klinik ergibt die 
Diagnose: Skrofulose. Patient wird in die Augenklinik aufgenommen. L. A. reizlos. Aussen am Hornhautrand eine zarte Trübung. S. $=1,0$. R. A. Bulbus lebhaft gerötet. Epithel der Cornea gestichelt, in ihr wolkige Trübungen, die im medialen Teil besonders dicht sind. Sie gehören den mittleren Schichten an. Von allen Seiten ziehen dichte, lange Gefässe durch die Hornhant. Iris soweit sichtbar hyperämisch. Kein roter Reflex vom Fundus. R. S. = Handbewegungen vor dem Auge. Ord. Atropin, Verband.

26. III. Wegen Verdacht auf Tuberkulose Probeinjektion von 0,0005 $\mathrm{ecm}$ Alt-Tuberkulin.

27. III. Keine Reaktion.

28. III. 0,001 cem Alt-Tuberkulin.

29. III. Deutliche allgemeine Reaktion.

1. IV. Patient fühlt sich wohl. $1 / 1000 \mathrm{mg}$ Bacillenemulsion.

13. IV. Da einmal mässig starke Reaktion auftrat, wurde sehr langsam gestiegen. Heute $5 / 1000 \mathrm{mg}$. Letzte Injektion waude gut vertragen. Auge völlig reizlos. Hornhaut etwas lichter.

10. V. Gestern $8 / 100 \mathrm{mg}$. Injektionen werden gut vertragen. Auge danernd reizlos, Hornhant wird glatt, spiegelnd. Im Zentrum eine lichtere Stelle. Pupille auf Atropin weit.

16. V. R. S. $=0,5$ !

11. VI. Patient erhält seit dem 1. VI. dauernd $1 \mathrm{mg}$, das gut vertragen wird. Auge dauernd reizlos. Epithel der Hornhant glättet sich immer mehr. Die Trübungen zerfallen, die Gefässe bilden sich jetzt rasch zurück. R. S. $=0,5-0,6$.

26. VI. Leichte Injektion. In der Hornhaut oben einige neue Gefässe, Zentrum der Hornhaut trüber. R. S. $=0,3-0,4$. Ord. weiter $1 \mathrm{mg}$.

2. VII. Auge wieder reizlos.

12. VII. Entlassung. Patient erhielt 50 Injektionen. Auge in letzter Zeit dauernd reizlos. Die Hornhaut spiegelt jetzt überall, hat sich hauptsächlich im Zentrum aufgehellt, nur noch vereinzelt ziehen Gefässe in die Cornea. Keine Beschläge an der Descemet. Kammerwasser klar. Iris reizlos. Fundus normal. R. S. $=0,5-0,6$. L. A. unverändert.

22. VII. Vorstellung, oben innen ein kleines frisches Infiltrat. Ord. Atropinsalbe.

2. VIII. Auge wieder blass. Ord. Vorstellung nach 2-3 Monaten. Patient wohnt auswärts, ist nicht erschienen (vgl. S. 251).

\section{Fall.}

Anna Sch., 20 Jahre alt. Eltern und Gesehwister sind gesund, auch Patientin war bis vor 3 Monaten gesund. Zu dieser Zeit schwoll das linke Oberlid an und wurde schwer. Zugleich rötete sich der Augapfel. Die Schwellung soll inzwischen etwas abgenommen haben. Patientin leidet zurzeit auch an geschwollenen Halslymphdrüsen.

22. V. 06. Der Aufnahmebefund ist folgender: R. A. normal. L. A. Oberlid ödematös geschwollen und etwas gerötet. Die Schwellung ist aussen stärker als innen. Das Lid hängt etwas herab. Das Unterlid ist äusserlich normal, die Bindehant aber etwas gerötet. Im Conjunctivalsack befindet sich etwas schleimiges Selkret. Beim Ektropionieren des Oberlids sielat 
man die ganze Übergangsfalte und die ganze Conj. tarsi bis auf einen kleinen Abschnitt innen dunkelrot verfärbt und von sagokorngrossen, trachomfollikelähnlichen Prominenzen dicht besetzt. Aussen sind diese Wucherungen wallartig um ein $5 \mathrm{~mm}$ langes, $2-4 \mathrm{~mm}$ breites, tiefes Geschwür angeordnet. Letzteres ist mit gelblichem Eiter bedeckt. Nach aussen von diesem Geschwür befindet sich noch oin zweites, das viel kleiner und flacher ist. Der Augapfel ist normal. R. S. $=1,0$. L. S. $=0,7$.

24. V. Da zweifellos Tuberkulose vorliegt, wird gleich mit ${ }^{1 / 1000} \mathrm{mg}$ Bacillenemulsion begonnen.

- $25 . \mathrm{V}$. Ein Stückchen Bindehaut wird in die Vorderkammer eines Kaninchens geimpft. Am 20. VI. werden bei dem Versuchstier zahlreiche Knoten in der Iris und ein hirsekorngrosser in der Sklera beobachtet.) -

14. VI. Mit den Dosen muss sehr langsam gestiegen werden, da dreimal mässig starke allgemeine Reaktion auftrat. Heute ${ }^{9 / 1000} \mathrm{mg}$. Schwellung des Lids ist geringer. Die Lidspalte ist normal. Der Wulst um das grosse Geschwür herum ist flacher, das Geschwür selbst seheint sich zu verkleinern.

21. VI. Gestern ${ }^{3} / 100 \mathrm{mg}$, hente Temp. $39^{\circ}$.

22. VI. Kein Fieber mehr.

23. VI. Wieder ${ }^{3} / 100 \mathrm{mg}$.

15. VII. Sehr langsam ist bis $5 / 10$ gestiegen. Injektionen werden jetzt gut vertragen. Oberlid schwillt rasch ab. Das grosse Geschwïr hat sich gereinigt und verkleinert.

25. VII. Pat. erhält dauernd $1 \mathrm{mg}$.

3. VIII. Injektionen werden dauernd gut vertragen. Das Oberlid schwillt immer mehr ab. Auch die Conjunctiva schwillt ab und wird blasser. Die Wucherungen sind flacher, zum Teil schon verschwunden. Das grosse Geschwuir ist nur noch stecknadelkopfgross. Das kleine Geschwür ist inzwischen abgeheilt.

16. VIII. Das Geschwür erscheint als kleine, gelbweisse Stelle.

10. IX. Da wenig Fortschritt zu bemerken ist, wird bei der 52 . Injektion auf $1,5 \mathrm{mg}$ gestiegen.

26. IX. Injektionen gut viertragen. Die letzten Wucherungen bilden sich sehr langsam zurick, werden daher einmal kauterisiert.

20. X. Entlassung. Erhielt 70 Injektionen. R. A. unverändert. L. A. Lider äusserlich wie rechts. Die grössere, mediale Hälfte der Bindehaut des Oberlids hat normales Aussehen, während die dunkelrote Färbung und intensive Schwellung des äusseren Teiles einer blasseren Farbe und leichthöckerigen Beschaffenheit Platz gemacht hat. Die Geschwüre sind verheilt. Die wallartige Verdickung um das Geschwür herum ist völlig verschwunden. An dieser Stelle sind nur noch leichte Prominenzen vorhanden. Sonst normaler Befund. $\mathrm{S} .=1,0$.

3. IV. 07. Vorstellung. Lider äusserlich normal. Aussen auf der Bindehaut des Oberlids, die nicht mehr geschwollen ist, einige kleine, graue Prominenzen und zwischen diesen ein kleiner Substanzverlust. Ord. Vorstellung.

29. V. Lider äusserlich normal. Prozess in der Bindehaut des linken Oberlids völlig ausgeheilt, nirgends mehr Prominenzen sichtbar. An Stelle der Geschwüre Narben. 


\section{Fall.}

Anna H., 15 Jahre alt. Familie ist gesund. Pat. litt im Jahre 1905 an schwerer Tuberkulose der Conjunctiva tarsi et bulbi rechts, die infolge Tuberkulinkur (T. R.) und operativer Behandlung heilte. Der Defekt wurde durch einen Epidermislappen gedeckt. (Am Versuchstier wurde seinerzeit schwere Tuberkulose der Iris erzeugt.)

Am 20. VI. 06. Wiederaufnahme wegen Rezidiv. R. A. Oberlid hängt herab, so dass die Pupille verdeckt ist. Im inneren Lidwinkel schleimiges Sekret. Der transplantierte Lappen ist selur gut angeheilt, nicht geschrumft. Es besteht ein guter Conjunctivalsack. Dort, wo der Lappen in die stark gerötete Conjunctiva des Lides übergeht, befinden sich mehrere stecknadelkopfgrosse Knötchen; 3 sitzen aussen an der Grenze des Lappens. Der Augapfel ist gut beweglich. Das untere Lid ist normal. Die obere Hornhauthälfte ist wie früher getrübt. Sonst normale Verhältnisse. L. A. normal.

21. VI. ${ }^{1 / 1000} \mathrm{mg}$ Bacillenemulsion.

15. VII. Injektionen werden gut vertragen, hente $\left.{ }^{4}\right\}_{400} \mathrm{mg}$.

Der Status ist wenig verändert, nur ist die Conjunctiva etwas blasser.

(Ein abgetragenes Knötchen wird in die vordere Angenkammer eines Kaninchens gebracht, worauf sich beim Versuchstier drei Knoten in der Iris bilden.)

16. VIII. Geringe Temperatursehwankungen abgerechnet, werden die Injektionen sehr gut vertragen. Noch immer leichte Sekretion. Conjunctiva blasser. Knötchen verkleinern sich sehr langsam.

Vom 20. VIII. bis 7. IX. ständig $1 \mathrm{mg}$ Bacillenemulsion. Injektionen werden gut vertragen. Geringe Besserung im Befunde.

Vom 9. IX. bis 17. X. danernd $1,5 \mathrm{mg}$. Diese Dosis wird reaktionslos vertragen.

26. IX. Die zwar zurïckgebildeten aber noch sichtbaren Knötchen werden mit dem Flachbrenner betupft. Die Kauterisation wird am 29. IX. und 4. X. wiederholt.

20. X. Entlassung. Patientin erhielt 50 Injektionen, sieht sehr wohl aus. R. A. im inneren Lidwinkel noch etwas Sekret. Das Oberlid verdeckt die Pupille nur noch zur Hälfte. Der transplantierte Lappen liegt unverändert gut. Die erhaltene Conjunctiva hat im ganzen eine normale, blassrote Farbe. Aussen sind in der Übergangsfalte noch einige höckerige Stellen sichtbar, die aber nicht wie Knötchen aussehen. Die Hornhaut ist unverändert. R. S. $=0,2$ bis 0,3 . L. A. unverändert, normal.

8. XII. Vorstellung. Aus den erwähnten, höckerigen Stellen haben sich kleine hahnenkammartige Wucherungen gebildet. (Diese Wucherungen bilden sich, wie wir weiter unten sehen werden, spontan zurück.)

Wenn wir uns das Wesentlichste aus diesen zehn Krankengeschichten nochmals vor Augen führen, so handelt es sich im ersten Fall neben einer alten. Chorioiditis um eine typische, tuberkulöse Iritis. Im Verlauf von 40 Injektionen Bacillenemulsion heilt die Regenbogenhautentzündung ab, und es bilden sich die Knötchen zurück. Die Injektionen werden gut vertragen. Patient nimmt während der Behandlung an Körpergewicht bedeutend $\mathrm{zu}$. 
Der zweite Fall zeichnet sich durch einen hervorragend chronischen Verlauf aus und dureh Hartnäckigkeit, da die Erkrankung trotz ärztlicher Behandlung acht Jahre lang nicht zur Ruhe kam. Im wesentlichen finden wir bei der Aufnahme eine sehr schwere tuberkulöse Iritis mit Knötchen im Kammerfalz, sodann Hornhauttrübungen und eine diffuse Trübung des Glaskörpers. Unter 50 Injektionen Bacillenemulsion wird die Regenbogenhaut atrophisch, die Knötchen bilden sich zurück, und der Glaskörper wird klarer. Zwei Monate nach der Entlassung sind die Knötchen vollständig verschwunden, nach einem weiteren Monat ist die Hornhaut vollständig klar und auch der Glaskörper hat sich so aufgehellt, dass das Sehvermögen von Erkennen von Fingern in $3 \mathrm{~m}$ auf 0,1 gestiegen ist. Die Injektionen, von ${ }^{1 / 1000} \mathrm{mg}$ beginnend, wurden anfangs sehr gut vertragen. Auf Dosen über ${ }^{1 / 10} \mathrm{mg}$ traten vor allem lokale Reaktionen auf, die jedoch die fortschreitende Besserung nicht hinderten. Als höchste Injektion wurde ${ }^{4} / 10 \mathrm{mg}$ schliesslich gut vertragen.

Im dritten Fall handelt es sich um eine schwere, tuberkulöse Iritis mit buckelartigen Vortreibungen der Iris und Knötchen im Kammerfalz, sodann auch um Infiltrate in der Cornea. Unter 41 Injektionen heilen die Infiltrate ab. Auch die Iritis heilt, Buckel und Knötchen verschwinden, und das Pupillarexsudat wird erheblich dünner. Das Sehvermögen steigt von Erkennen von Fingern in $3 \mathrm{~m}$ auf $\mathrm{S} .=0,3 . \mathrm{Da}$ nach der Bacillenemulsion oft Reaktionen auftraten, wurden zum Schluss fünf sehr schwache Injektionen T. R. gemacht. Auch auf T.R. erfolgte eine allgemeine Reaktion. Übrigens schadeten die Reaktionen dem Patienten in keiner Weise. Der Heilverlauf schritt am Auge beständig fort und Patient fühlte sich, abgesehen von der Zeit, wo Reaktionen bestanden, sehr wohl und nahm auffallend an Körpergewicht $\mathrm{zu}$.

Der vierte Fall zeichnet sich wieder durch einen eminent chronischen Verlauf und Hartnäckigkeit aus. Im wesentlichen handelt es sich bei der Aufnahme um einen Knoten in der Hornhaut und um eine schwere Iritis mit hinteren Synechien und einem Pupillarexsudat. Unter 54 Injektionen Bacillenemulsion, von ${ }_{1500} \mathrm{mg}$ bis $1 \mathrm{mg}$ steigend, bildet sich der Hornhautknoten unter Hinterlassung einer ganz feinen Trübung zurück, die Iritis heilt ab und das Pupillarexsudat verschwindet, so dass das Sehvermögen von Erkennen von Fingern in $2 \mathrm{~m}$ auf $\mathrm{S} .=0,7-0,8 !$ steigt. Die Injektionen wurden gut vertragen.

Bei der fünften Patientin finden wir neben alten Veränderungen 
frische episklerale Knötchen und Infiltrate der Hornhaut mit Iritis. Da nach der Probeinjektion dentliche lokale und allgemeine Reaktion auftrat, so wurde mit $1 / 1000 \mathrm{mg}$ Bacillenemulsion begonnen. $60 \mathrm{In}-$ jektionen - zuletzt dauernd $1 \mathrm{mg}$ - werden gut vertragen. Die episkleralen Knötchèn verschwinden, die Infiltrate heilen $a b$, ebenso die Iritis. (Der Visus blieb wegen der alten Aderhautveränderungen derselbe:)

Beim sechsten Fall kam zunächst das T. R. zur Anwendung. Die Tuberkulose der Cornea, verbunden mit Tritis, ging - wie früher - prompt zurück. Da nach T. R. einmal Reaktion auftrat und zur Vermeidung von Rezidiven, wurde bei den letzten Injektionen die Bacillenemulsion angewandt. Die Heilung schritt unter der Behandlung mit Bacillenemulsion, die vorziiglich vertragen wurde, gut fort. Im Verlauf der ganzen Behandlung stieg das Sehvermögen von 0,1 auf $0,3-0,4$; ein $\mathrm{Jahr}$ später betrug es $\mathbf{S} .=0,6$ !

Im siebenten Fall handelt es sich um eine chronische Keratitis, die unter keiner Behandlung zur Ruhe kommen wollte. Die Probeinjektion bestätigte den Verdacht auf Tuberkulose. Es wird mit $1_{1000} \mathrm{mg}$ Bacillenemulsion begonnen. Schon nach wenig Injektionen wird das Auge reizlos. Ein zarter Pannus, der im Verlauf der Behandlung entsteht, bildet sich zurück. Injektionen über ${ }^{1} / 10 \mathrm{mg}$ werden anfangs nicht gut vertragen. Patient erhält im ganzen 50 Injektionen, nimmt an Körpergewicht zu. Das Sehvermögen, das bei der Aufnahme infolge starker Lichtscheu nicht zu bestimmen war, betrug bei der Entlassung S. $=0,1$. Ein kleiner Pannus, der sich wenige Tage nach der Entlassung wieder bildete, heilte rasch ab. Laut Bericht nach sechs Monaten war das Auge reizlos.

Im achten Fall haben wir eine parenchymatöse Keratitis auf dem rechten Auge. Die Anamnese spricht für Tuberkulose, die Probeinjektion verläuft positiv. Injektionen von $1_{1000}$ bis $1 \mathrm{mg}$ Bacillenemulsion werden gut vertragen. Das Auge ist rasch reizlos, und die Hornhaut hellt sich soweit auf, dass das Sehvermögen von Erkennen von Handbewegungen vorm Auge auf $0,5-0,6$ ! steigt.

In den beiden letzten Fällen handelt es sich um typische Tuberkulose der Bindehaut, die auch durch Tierversuch bestätigt wird. In dem einen Fall finden wir zahlreiche trachomähnliche Follikel und zwei Geschwüre. Die Injektionen werden mit $1 / 1000 \mathrm{mg}$ Bacillenemulsion begonnen und werden bis $1,5 \mathrm{mg}$ gesteigert. Die Geschwüre heilen $a b$, und die Wucherungen bilden sich zurück. Da einzelne sehr langsam schwinden, werden sie einmal kauterisiert. Nach 70 Injektionen, 
die gut vertragen wurden, wird die Patientin entlassen. Nach sieben Monaten ist die Conjunctiva normal bis auf Narben an Stelle der Geschwüre.

In dem zweiten Fall von Conjunctivaltuberkulose handelt es sich um ein Rezidiv. Unter 50 Injektionen Bacillenemulsion, die gut vertragen werden, bilden sich die Knötchen zurück, zur vollständigen Heilung ist jedoch ein dreimaliges Betupfen mit dem Thermokauter notwendig. Bei der Entlassung sieht die Conjunctiva normal aus, nur aussen auf der Übergangsfalte sind noch einige höckerige Stellen, die aber nicht den Eindruck von Knötchen machen. Nach sechs Wochen sind an diesen Stellen kleine hahnenkammartige $W$ ucherungen sichtbar, die sich jedoch spontan zurückbilden, wie wir weiter unten sehen werden.

Die Resultate, die in vorliegenden Fällen mit der Bacillenemulsion erzielt wurden, sind als ausserordentlich günstige zu bezeichnen. In keinem Fall liess das Präparat im Stich, auch nicht bei der Tuberkulose der Bindehaut, wenn auch bei dieser zur Abkürzung bzw. zum endgültigen Abschluss des Heilverfahrens die Kauterisation notwendig wurde. Was mir an den Resultaten besonders bemerkenswert erscheint, ist folgendes: Bei den verschiedenen Formen von Keratitis hellte sich die Hornhaut auffallend auf. Im zweiten Fall schwanden die Trübungen der Hornhaut gänzlich, im vierten Fall blieb nach einem dichten Knoten nur eine ganz feine Trübung zurück, bei Fall 6 und 8 hellte sich die Cornea soweit auf, dass der Visus von 0,1 auf 0,6 bzw. von Erkennen von Handbewegungen vorm Auge auf 0,5 bis 0,6 stieg!

Sodann scheint mir besonders erwähnenswert, dass in allen unsern Fällen von Tuberkulose der Iris die Knötchen prompt schwanden, auch dann, wenn sie zunächst noch Tendenz zur Vermehrung bzw. Vergrösserung zeigten. Auch die Pupillarexsudate bildeten sich auffallend gut zurück, wodurch in Fall 4 sich das Sehvermögen von Erkennen von Fingern in $2 \mathrm{~m}$ auf $\mathrm{S} .=0,7-0,8$ hob. Bei Fall 2 scheint mir die Aufhellung des Glaskörpers besonders bemerkenswert zu sein, auch die rasche Rückbildung der episkleralen Knötchen im Fall 5 möchte ich hier hervorheben, denn auch diese vermehrten sich zunächst noch unter der Beobachtung.

Erfahrungen über die Einwirkung der Bacillenemulsion auf Erkrankungen der Aderhaut konnten nicht gesammelt werden, da nur alte Fälle von Chorioiditis als Nebenbefunde beobachtet wurden. 
Recht würdigen kann man diese Resultate, wenn man sie mit denen vergleicht, die frither erzielt wurden. Es ist bekannt, dass, bevor das Tuberkulin von Koch eingeführt wurde, die Erfolge bei der Behandlung der Augentuberkulose schlecht waren. Trotz aller Bemühungen gingen damals viele Augen zugrunde. Wie gross die Verluste früher waren, ersieht man deutlich aus einer im Jahre 1900 von Schieck (1) veröffentlichten Statistik. Unter 116 Fällen von Iristuberkulose, die Schieck aus der Literatur sammelte, fand er 64 Verluste.

Schlecht waren die Erfolge vor allem bei schwerer Tuberkulose der Augen. Ich glaube, dass auch unter unsern Fällen mehrere Augen erblindet bzw. zugrunde gegangen wären ohne die Tuberkulinkur, denn es befinden sich unter ihnen doch auch einige recht schwere Erkrankungen. Auch von jenen Fällen, die auf den ersten Blick als leichte Formen angesprochen werden, müssen einige zu den schweren gerechnet werden, da sie zu Beginn der Behandlung noch deutliche Zeichen des Fortschreitens der Erkrankung trugen. Gegenüber jener Zeit, wo das Tuberkulin noch nicht verwandt wurde, sind wir heute fraglos sehr im Vorteil. Denn unter unsern Fällen befinden sich überhaupt keine Verluste, sondern nur prompte Heilerfolge. Eine andere Frage ist, ob die Bacillenemulsion mehr leistet als die andern, älteren Präparate Kochs. Vom Alttuberkulin will ich absehen, da dieses in der Ophthalmologie fast nur noch zu diagnostischen $Z_{\text {wecken, }}$ wo es allerdings von grösstem Werte ist, verwandt wird. Es bleibt also nur ein Vergleich mit dem T. R. übrig. Wenn auch die bier veröffentlichten, mit der Bacillenemulsion behandelten Fälle nicht sehr zahlreich sind, so sind doch die Erfolge so gleichmässig gute, dass ich die Bacillenemulsion dem T. R. ebenbürtig erachte. Entscheidend ist für die Beantwortung dieser Fragejedoch, welches von beiden Präparaten am sichersten vor Rezidiven schützt. Denn, wie oben erwähnt, ging v. Hippel nur deshalb zur Bacillenemulsion über, weil er nach dem T. R. wiederholt Rezidive beobachtet hatte. Wir müssen nun sehen, ob die Heilungen bei der Bacillenemulsion wirklich dauerhaftere waren, was wir nach dem Urteil Kochs erwarten mussten. Nachfragen bzw. Nachuntersuchungen bei unsern zehn Fällen, die Herr Dr. Thies, Assistenzarzt der Universitäts-Augenklinik zu Göttingen, in liebenswürdiger Weise übernahm, haben nun folgendes ergeben:

1. Ernst K. Entlassen am 5. XII. 06. Die letzte Untersuchung am 25. VI. 08 ergab: Die drei Knötehen der Iris sind verschwunden. Beiderseits $\mathrm{S} .=1,0$. 
2. Paula W. Entlassen am 5. IV. 07. Iretzte Untersuchung Ende April 08: R. A. unverändert. L. A. danernd reizlos, äusserlich völlig unverändert. Glaskörper vielleicht etwas träber ${ }^{1}$ ). L. S. = Erkennen von Fingern in $3 \mathrm{~m}$.

$\left.\begin{array}{l}\text { 3. Julius S. } \\ \text { 4. Frau H. }\end{array}\right\}$ Nicht zu ermitteln.

5. Christine E. Entlassen am 28. III. 07. Die letzte Untersuchung Ende Juni 08 ergab: Kein Rückfall. Patientin fühlt sich sehr wohl. Der Visus ist links auf 0,1 gestiegen.

6. Marie K. Entlassen am 9. V. 06. Laut Nachricht vom 29. V. 08 trat Mitte März 08 ein Rückfall ein, der unter erneuter Tuberkulinkur gut zurüekging.

7. Gust. R. Entlassen am 10. XI. 06. Laut Nachricht vom 29. VI. 08 ist dauernde Heilung eingetreten.

8. Heinr. H. Entlassen am 12. VII. 07. Am 29. VI. 08 Nachricht rom Vater: 6 Monate nach der Entlassung soll eine leichte Entzündung von kurzer Dauer ${ }^{2}$ ) anfgetreten sein. Seitdem dauerndes Wohlbefinden.

9. Anna S ch. Entlassen am 20. X. 07. Untersuchung am 1. VII. 08 ergibt, dass Patientin danernd geheilt ist und sich körperlich sebr wohl fühlt.

10. Anna H. Entlassen am 20. X. 06. Die letzte Untersuchung am 4. VII. 08 ergab: Keine verdächtigen Stellen. Die kleinen, hahnenkammartigen Wucherungen haben sich zurückgebildet. Patient fühlt sich sehr wohl.

Die Beobachtungszeit schwankt zwischen 1-2 Jahren. Der einzige sichere Rückfall trat bei Frau K. ein. Dies ist sehr auffallend, da in diesem Fall (Fall 6), als dem einzigen, im wesentlichen das T. R. zur Anwendung kam. Nur bei den letzten sieben Spritzen wurde versuchsweise die Bacillenemulsion gegeben. Da nach so wenig Injektionen ein dauernder Erfolg nicht erwartet werden kann, so lege ich diesen einzigen Rückfall nicht der Bacillenemulsion, sondern dem T. R. zur Last. Über zwei Patienten konnte nichts in Erfahrung gebracht werden. Zu den übrigen Fällen brauche ich nichts hinzuzufügen, sie sprechen für sich selbst und beweisen, dass bei allen diesen Patienten Rezidive nicht auftraten, dass sogar in mehreren Făllen noch nachträglich erhebliche Besserung konstatiert werden konnte.

So hat sich denn bisher die Hoffnung erfüllt, die wir nach der Mitteilung $K$ ochs auf die Bacillenemulsion setzen mussten, dass sie nämlich mehr als das T.R. vor Rezidiven

1) Vielleicht hat auch die Trübung der Linse etwas zugenommen.

2) Der Vater des Patienten schreibt so dankbar und zufrieden, dass es sich wohl nicht um einen Rückfall gehandelt haben wird, sondern um einen leichten, zufälligen Reizzustand. 
schützt. Da sie auch sonst, wie wir gesehen haben, keinerlei Nachteile dem T. R. gegenüber besitzt, so glaube ich bei der Behandlung der Tuberkulose des Auges der Bacillenemulsion vor dem T.R. den Vorzug geben zu müssen.

Ich hatte die Absicht, durch diese Arbeit die Aufmerksamkeit auf ein Präparat zu lenken, das nach unserer Erfahrung bisher nicht genügend gewürdigt wurde. Ich hoffe, dass die durchweg guten, dauerhaften Resultate, die mit der Bacillenemulsion an der Universitäts-Augenklinik zu Göttingen erzielt wurden, zu weitgehender Anwendung dieses Präparats bei der Behandlung tuberkulöser Augenerkrankungen ermutigen werden.

Bemerken will ich noch, dass die Bacillenemulsion viel billiger ist als das T. R. In gleicher Originalpackung kostet die Bacillenemulsion nur 1,25 Mk., während der Preis für das T. R. 8,50 Mk. beträgt.

Ich erfülle eine angenehme Pflicht, wenn ich meinem früheren Chef, Herrn Geheimrat v. Hippel, herzlichst für das liebenswürdige Interesse danke, das er dieser Arbeit entgegenbrachte.

\section{Literaturverzeichnis.}

1) Schieck, Klinische und experimentelle Studien über die Wirkung des Tuberkulins auf die Iristuberkulose. v. Graefe's Arch. Bd. L, 2. 1900.

2) v. Hippel, A., Uber den Nutzen des Tuberkulins bei der Tuberkulose des Auges. v. Graefe's Arch. Bd. LIX, 1. 1904.

3) Fleischer, Demonstration zweier Fälle ron Conjunctivaltuberkulose. Ophth. Klinik. 1905. S. 212.

4) Gilbert, Zur Prognose und Therapie der Conjunctivaltuberkulose. Klin. Monatsbl. f. Augenheilk. Bd. II. S. 22. 1905.

5) Lundsgaard, Om Tuberculosis conjunctivae og dens Behandling. Hospitalstidende. No. 39,42 u. 43 . p. 805.1905.

6) Schwarz, Heilung eines Falles von Tuberkulose der Bindehaut durch Marmoreks Serum. Deutsche med. Wochenschr. Nr. 34. 1905.

7) Wolfrum, Fünf Fälle von Tuberkulose des Auges unter Behandlung mit Tuberkulin T. R. Arch. f. Augenheilk. Bd. LIV. S. 1. 1906.

8) Brückner, Erfahrungen mit dem Kochsehen Tuberkulin (Alt-Tuberkulin und Tuberkulin T. R.). Arch. f'. Augenheilk. Bd. LVI. S. 352. 1906.

9) Elsaesser, Űber die Behandlung der Tuberkulose mit Marmorekserum und Nen-Tuberkulin (Bacillenemulsion) nebst einigen Ausblicken in die Zukunit der Tuberkulosebekämpfung. Deutsche med. Wochenschr. Nr. 51. 1907.

10) Reis, Primäre Tuberkulose der Conjunctiva. Klin. Monatsbl. f. Augenheilk. 1907. Neue Folge 3. S. 158.

11) Stock, Tuberkulose als Ätiologie der chronischen Entzïndungen des Auges und seiner Adnexe, besonders der chronischen Uveitis. จ. Graefe's Arch. Bd. LXVI, 1. 1907.

12) v. Hi p pel, A., Weitere Erfahrungen über die Behandlung schwerer Tuberkulose des Auges mit Tuberkulin. 32. Ophth. Vers. Heidelberg 1905. S. 136. 
13) Capolongo, Tuberculosi della conjunetivt. Contributo elinico anatomico e sperimentale. Annali d'Ottalm. XXXV. p. 933. 1906.

14) Rohmer, Trois cas de Kératite parenchymateuse traitée par la tuberculine T. R. Revue générale d'Opht. 1906. p. 554.

15) Wilder, Tuberculosis of the cornea. Ophth. Record. p. 610. 1906.

16) Bulson, Further report on the successful use of tuberculin in a case of iritis tuberculosa, Journ. of the Americ. Med. Assoc. 1905.

17) Gamble and Brown, Further report on the case of iritis tuberculosis as diagnosed and treated by Koch's tuberculin. Joum. of the Americ. Med. Assoc. 1905 . Oct. 14.

18) Laspeyres, Beiderseitige Iristuberkulose. Dentsche med. Wochenschr, 1906. S. 2010 .

19) Lundsgaard, Behandlung von Lupus conjunctivae. Klin. Monatsbl. f. Augenheilkunde. 1906. S. 191.

20) Reuchlin, Uber Erfahrungen mit dem Kochschen Tuberkulin. Klin. Monatsbl. f. Augenheilk. Bd. XLIV. S. 352, 1906.

21) Erdmann, Über eine erfolgreiche Behandlung von Tuberkulose des Auges mit Tuberkulin. Münch. med. Wochenschr. Nr. 14. 1907.

22) Ruppel, Die Herstellung des ,neuen Tuberkulins" (Tuberkulin T. R.). (Eine Berichtigung.) Deutsche med. Wochenschr. Nr. 5. 1908. 